\title{
Nasopharyngeal Rhabdomyosarcoma: A Rare Malignancy Incidentally Found in a Middle-Aged Male with a Diagnostic Dilemma
}

\author{
Authors: \\ *Muhammad Sohaib Asghar, ${ }^{1}$ Mariam Amir,, Hiba Shariq,, ${ }^{1}$ Narmin \\ Khan, ${ }^{1}$ Maira Hassan, ${ }^{2}$ Rumael Jawed, ${ }^{2}$ Uzma Rasheed, ${ }^{2}$ Faran Khalid \\ 1. Dow University Hospital, Dow University of Health Sciences, Karachi, Pakistan \\ 2. Liaquat National Hospital \& Medical College, Karachi, Pakistan \\ *Correspondence to sohaib_asghar123@yahoo.com
}

Disclosure: $\quad$ The authors have declared no conflicts of interest.

Acknowledgements: Ethical approval for this study was provided by an institutional review board, and consent to participate has been taken from the patient's guardian with informed verbal consent.

Received:

09.05 .20

Accepted:

13.07.20

Keywords:

Chemotherapy, immuno-histochemistry, nasopharynx, poorly differentiated, rhabdomyosarcoma (RMS).

Citation:

EMJ Oncol. 2020;8[1]:122-128.

\section{Abstract}

Nasopharyngeal rhabdomyosarcoma is a rare tumour of the paediatric age group that emerges from embryonal mesenchymal cells. Presented here is a case of a 54-year-old male of Asian ethnicity with a notable history of weight loss, lack of energy, anal fissure, and haematochezia. After the incidental finding of a lytic lesion following imaging, the patient underwent an extensive work-up to rule out malignancy and increased uptake on nasopharynx was found, which was biopsied to diagnose a poorly differentiated tumour, having desmin and myogenin positivity on immunohistochemistry. Metastatic work-up showed extensive bone marrow invasion apart from multiple lytic bone lesions throughout the body. The patient was started on vincristine, actinomycin D (dactinomycin), and cyclophosphamide (VAC) protocol chemotherapy and was followed-up until two cycles were completed, with no evidence of disease remission.

\section{INTRODUCTION}

Rhabdomyosarcoma (RMS) is an infrequent and aggressive malignancy that emerges from embryonic mesenchymal cells all around the body, including sites devoid of striated muscles. ${ }^{1}$ RMS has an unknown aetiology as genetics, chemical hazards, viruses, and environmental factors have all been considered a cause of pathogenesis. ${ }^{2}$ In 1958, Horn and Enterline classified RMS into four histological classifications: alveolar, pleomorphic, embryonal, and botryoid. The embryonal subtype is the most frequent in children (50-60\%), the alveolar subtype is the most common subtype seen in adolescents until the age of 25 , and the pure pleomorphic subtype occurs merely in adults. ${ }^{3}$ RMS is the third most common extracranial tumour in children after neuroblastoma and Wilms' tumour, respectively, and frequently arises within the nasal cavity 
and paranasal sinuses of the paediatric population. ${ }^{4}$ Incidence per annum of RMS in children is reported at 4.3 cases per million. ${ }^{1}$ It follows a bimodal distribution in the general population, with peak occurrences between 2 and 4 years and 12 and 16 years, respectively. ${ }^{5}$ RMS demonstrates a significant predisposition for Caucasians and commonly occurs in males. ${ }^{6}$ Comparatively, RMS occurs less frequently in adults. ${ }^{1}$ Soft tissue sarcomas constitute $<1 \%$ of all adult malignancies, and RMS accounts for $3 \%$ of all soft tissue sarcoma.7 Adult RMS do not show a propensity for males, as observed in paediatric patients, and occur primarily in the extremities. ${ }^{3}$ Furthermore, its occurrence in adults in the head and neck area is extremely rare. ${ }^{4}$ RMS of the head and neck region is divided into three perceptible groups: the orbital group, parameningeal group, and other head and neck location group.7 Among the parameningeal group, the nasopharynx is the most commonly reported site. ${ }^{7}$ Parameningeal lesions have the worst prognosis because of the difficulty in diagnosis; associated complications, i.e., central nervous system involvement; and operative complexity. ${ }^{7}$

\section{CASE PRESENTATION}

A 54-year-old male of Asian ethnicity presented with a notable history of weight loss, lack of energy, feverish feeling for almost 8 months, and complaints of lower back pain, fever, and generalised weakness for 1 month. He denied any cough, haemoptysis, abdominal pain, alternating bowel habits, melaena, haematochezia, urinary incontinence, urinary dribbling, hesitancy, flank pain, or haematuria. On further inquiry, he was noted to have occasional nasal blockage and rhinitis, and now has predominant complaints of haematochezia. He described months of episodic, severe pain on defecation associated with small volumes of fresh blood per rectum. Given the history, he was managed with antipyretics, nutritional supplements, a high fibre diet, hip baths, and topical ointments keeping the probability of anal fissure in view.

Physical examination results were considered normal, except that the patient was febrile with a temperature of $99^{\circ} \mathrm{F}$, and systemic examination was also unremarkable with no lymph nodes palpable and no bone tenderness reported. Ears, nose, and throat examination showed a deviated nasal septum towards the left side and rectal examination was deferred by the patient because of severe pain in the perineum; therefore, an MRI scan of the pelvis was recommended. Laboratory work-up revealed a haemoglobin level of 9.8 $\mathrm{g} / \mathrm{dL}$; total leukocyte count of 10.1 cells $/ \mu \mathrm{L}$, with a neutrophil count of $54 \%$ and lymphocyte count of $39 \%$; mean cell volume of 92 ; platelet count of 46 cells/ $\mu \mathrm{L}$; erythrocyte sedimentation rate of $120 \mathrm{~mm} /$ hour; C-reactive protein level of $52 \mathrm{~g} / \mathrm{dL}$; serum ferritin level of 2,000 ng/mL; lactate dehydrogenase level of 1,091 international units/L; creatinine phosphokinase level of 266 international units/L; serum creatinine level of $0.9 \mathrm{mg} / \mathrm{dL}$; and normal serum immunofixation and serum protein electrophoresis results. The differential considerations included initially were Pott's disease, multiple myeloma, Crohn's disease, or underlying malignancies including colorectal cancer, lymphoma, and chronic lymphocytic leukaemia.

The pelvis MRI ruled out perianal abscess, fistula, and intestinal mass and provided a diagnosis of anal fissure, but also showed multiple infiltrative Iytic bone lesions in the sacrum, acetabulum, and lumbar and sacral vertebrae (Figure 1). An immediate skull X-ray (Figure 1) and lumbosacral spine X-ray were also carried out, which showed punched out lesions within the skull, but the lumber and sacral spines appeared normal (Figure 2). Pott's disease was attributable to the patient's history of lower back pain, weight loss, fever, generalised weakness, and raised erythrocyte sedimentation rate, but it was ruled out on radiological imaging. The patient was managed conservatively for anal fissure, and further work-up for malignancy was planned after ruling out multiple myeloma. Further workup included urine Bence Jones protein and bone marrow biopsy; there was no trace of Bence Jones proteinuria. PET-CT demonstrated a mass in the right nasopharynx extending to the right maxillary sinus and a metastatic right cervical lymph node, and further revealed increased uptake in the thoracic lumbar vertebrae, sacrum acetabulum, right scapula, and the left $10^{\text {th }}$ rib. Biopsy of the nasopharyngeal mass exhibited RMS of anaplastic (undifferentiated) variety with immunohistochemical stains positive for desmin and myogenin (Figure 3). 


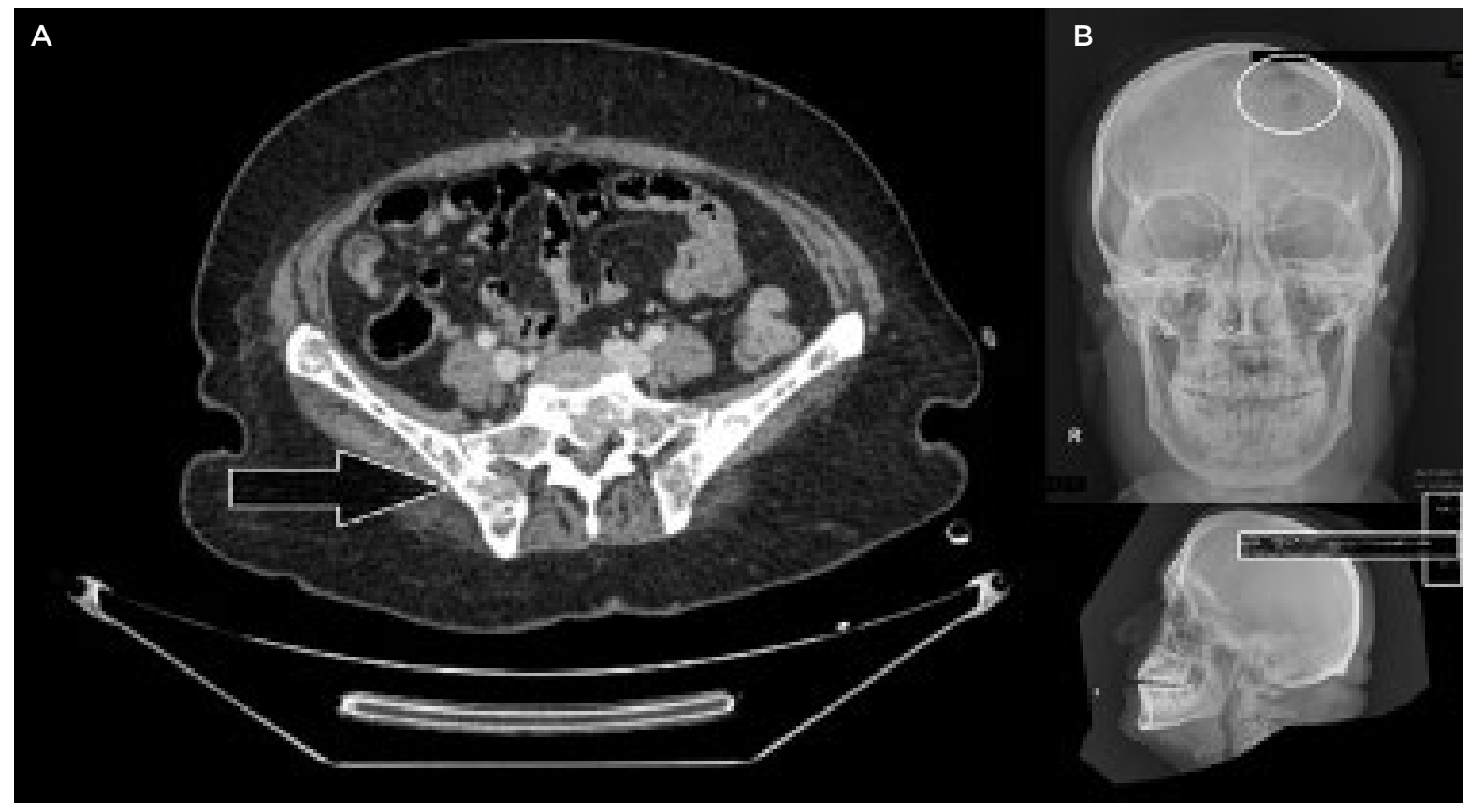

Figure 1: Lytic bone lesions over the pelvic girdle (A) and skull (B).

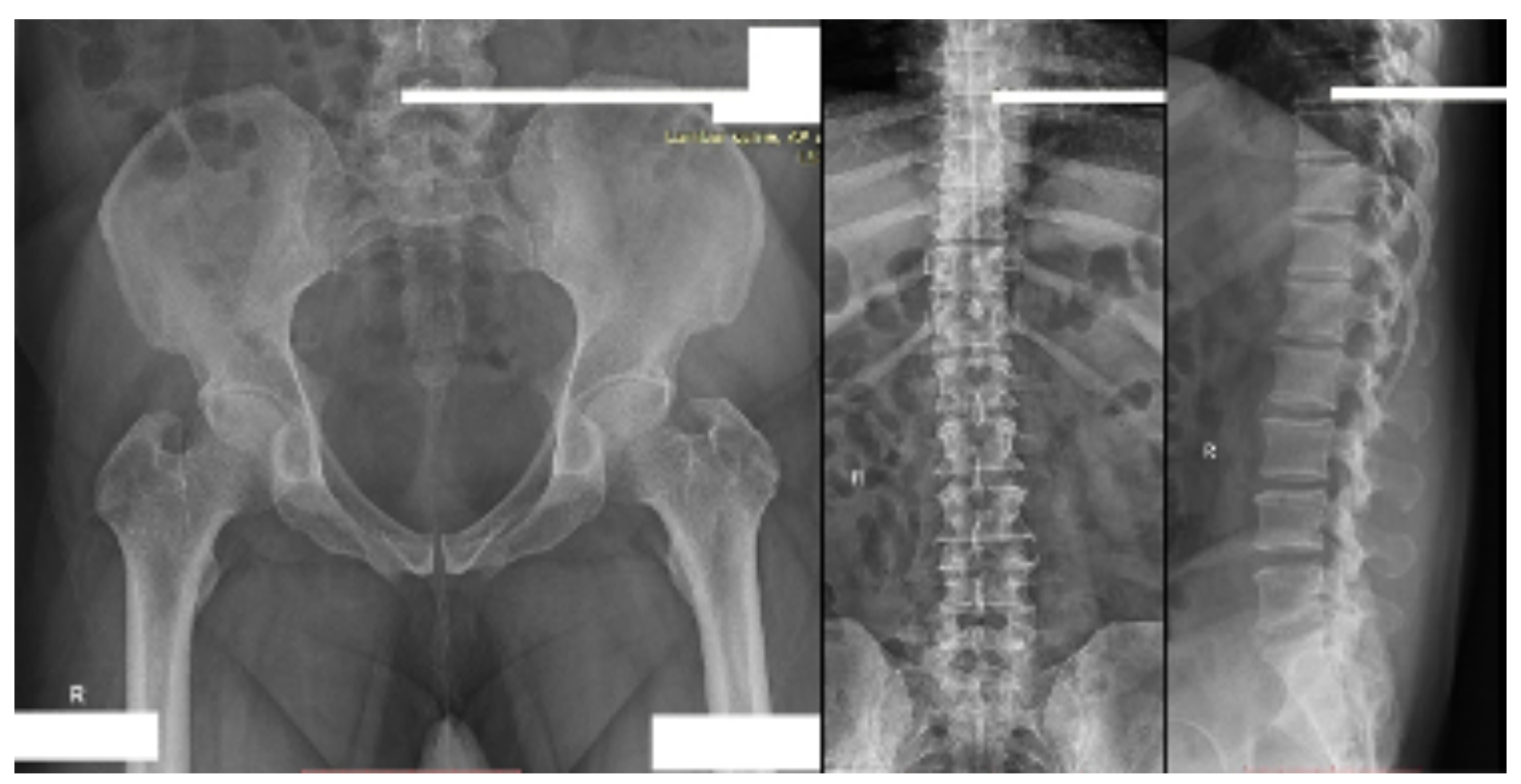

Figure 2: Normal lumbosacral spine and pelvic X-ray.

The histopathological differential diagnosis included small round blue cell tumours and pleomorphic sarcomas. Because of the weak positivity of CD117 and placental alkaline phosphatase, and to exclude a possibility of germ cell neoplasm (another differential diagnosis), a large panel of immunohistochemical stains were used; however, because of the nasopharyngeal location of the tumour and crisp positivity of desmin and myogenin, the diagnosis of embryonal RMS was made. Bone marrow biopsy was conclusive for metastatic infiltration, exhibiting a hypocellular specimen and clumps of non-haematopoietic cells. 
Gross: Specimen is received in a single container as "Nasopharyngeal mass" specimen comprising multiple, irregular, greyish, soft tissue pieces that collectively measure $1.0 \times 1.0 \times 0.6 \mathrm{~cm}$ in aggregate. Entirely submitted in a single cassette.

Microscopic features: Sections examined revealed multiple polypoidal fragments of respiratory mucosa covered by stratified squamous columnar ciliated epithelium and shows patchy areas of surface ulceration. Underlying tissue mucous secreting glands mixed inflammatory infiltrate. At places foci of neoplastic lesion are present. Cells are arranged in trabeculae. The cells are shown to have moderate amounts of cytoplasm, nuclei show moderate to marked pleomorphism with coarse chromatin and inconspicuous nucleoli. Special stains for glycogen (periodic acid-Schiff +/- diastase) is positive.

The sections were stained with the panel of the following immunohistochemical stains and neoplastic cells showed the following reactivity pattern:

\begin{tabular}{|l|l|}
\hline CD20 & negative \\
\hline CD3 & negative \\
\hline CKAE1/AE3 & negative \\
\hline S100 & negative \\
\hline Tdt & negative \\
\hline CK5/6 & negative \\
\hline CK7 & negative \\
\hline P4O & negative \\
\hline CK8/18 & negative \\
\hline Cyclin D1 & negative \\
\hline MUM 1 & negative \\
\hline Melan A & negative \\
\hline Desmin & strong positive \\
\hline Myogenin & strong positive \\
\hline CD117 & weak positive \\
\hline PLAP & weak positive \\
\hline LCA & negative \\
\hline
\end{tabular}

\begin{tabular}{|l|l|}
\hline CD4 & negative \\
\hline CD8 & negative \\
\hline CD34 & negative \\
\hline CD138 & negative \\
\hline CD30 & negative \\
\hline Synaptophysin & negative \\
\hline Chromogranin & negative \\
\hline CD68 & negative \\
\hline MPO & negative \\
\hline ASMA & negative \\
\hline Caldesmon & negative \\
\hline Oct 34 & negative \\
\hline CD56 & negative \\
\hline EMA & negative \\
\hline CD99 & negative \\
\hline Alk protein & negative \\
\hline P63 & negative \\
\hline Cytokeratin & negative \\
\hline
\end{tabular}

Conclusion: Nasopharyngeal mass: biopsy

Morphological and immunohistochemical features are in favour of rhabdomyosarcoma.

Figure 3: Nasopharyngeal mass biopsy immunohistochemistry report.

A large panel of immunohistochemical stains were used. Owing to the weak positivity of CD117 and placental alkaline phosphatase, germ cell neoplasm was also considered in this case; however, because of the nasopharyngeal location of the tumour and crisp positivity of desmin and myogenin, the overall features were in favour of rhabdomyosarcoma. Strong clinical and radiological correlation was advised.

According to the TNM classification, the tumour was classified as T3 (tumour grown into the sinuses and/or bones nearby), N2 (spread to nearby lymph nodes), and M1 (distant metastasis), stratifying the patient to Stage 4 disease. The patient was referred to the oncology department for palliative chemotherapy and was started on the VAC protocol chemotherapy regimen for metastatic RMS (vincristine 1.4 $\mathrm{mg} / \mathrm{m}^{2} /$ dose, dactinomycin $1.5 \mathrm{mg} / \mathrm{m}^{2} /$ dose, cyclophosphamide $1,500 \mathrm{mg} / \mathrm{m}^{2}$ ) for every 21 days cycle. ${ }^{8,9}$ 
After two cycles, the patient attended a followup visit with the disease still progressing and a haemoglobin level of $11.5 \mathrm{~g} / \mathrm{dL}$; total leukocyte count of $4.5,60 \%$ neutrophils and $27 \%$ lymphocytes; and a platelet count of 38 cells/ $\mu \mathrm{L}$. The follow-up period was limited because of the patient's ongoing chemotherapy. After two sessions of chemotherapy, the patient was lost to follow up.

\section{DISCUSSION}

With the advent of immunohistochemistry, electron microscopy, and molecular genetic studies, the histological diagnosis of RMS has remarkably ameliorated. ${ }^{3}$ Using these techniques, the tumour cells of the RMS express desmin, muscle-specific actin, and myoglobin in welldifferentiated tumour cells. ${ }^{3}$ Poorly differentiated tumour cells do not stain these agents; here vimentin was strongly positive. Other newly used markers include myoblast determination protein 1 and myogenin antibodies, as was the case in the described patient.

Regardless of the age and gender of a patient with RMS, the signs and symptoms depend on the tumours' origin and its invasion into abutting structures. $^{3}$ The time between the onset of symptoms and diagnosis averages from 1 week to 9 months. ${ }^{2}$ While superficial tumours may be asymptomatic or present with a tender mass, deep tumours cause vague symptoms and often significantly increase in size before being brought to medical recognition. ${ }^{5}$ Tumours arising in the paranasal sinuses, nasal cavities, mastoid, and nasopharynx present with symptoms of nasal obstruction, rhinorrhoea, and recurrent otitis media. ${ }^{5}$ Paranasal sinuses are the most common primary site. 10,11 RMS of sinus and nasal origin in adults present with local pain, epistaxis, nasal obstruction, otorrhea, deafness, and sinusitis, and advanced cases usually present with cranial nerve palsies.3,12 Symptoms of ophthalmoplegia and decreased vision as a result of direct invasion of the orbital apex have been reported in cases of nasopharyngeal RMS.12 Microscopically, these tumours appear pink, fleshy, and soft, while no variation exists between types except for the botryoidal variant. ${ }^{10} \mathrm{RMS}$ is equivalent to teratoma, as microscopic tissues present evidence of cartilage, bone, and other bodily tissues. ${ }^{13}$ Microscopically, the embryonal type shows increased cellularity, containing several undifferentiated mesenchymal cells and the presence of myxomatous changes. ${ }^{4}$ In contrast, the alveolar type shows small circular rhabdomyoblasts, arranged in nests or cards, separated by connective tissue trabeculae and focal locations of alveolar architecture. ${ }^{4}$ Metastasis via intracranial spread or to distant locations is the most common cause of death. ${ }^{14}$

RMS are high-grade tumours with local belligerence and a strong propensity to metastasise; hence, are considered a 'systemic disease' given the swift development of metastatic spread. ${ }^{5}$ RMS metastasises through direct tissue invasion, a haematogenous route, and by involving the lymphatic system. ${ }^{6}$ They differ from other forms of sarcomas by showing an increased predilection to metastasise via lymphatic channels..$^{13}$ RMS of the palate spreads to the deep cervical nodes, causing them to have a rubbery consistency, while atrophy and central necrosis tend to occur in the larger metastatic nodes. ${ }^{15}$ Nasopharyngeal RMS tends to grow rapidly and infiltrate the skull base or central nervous system. ${ }^{14}$ The direct invasion route poses a distinctive danger to the meninges, especially when the tumour inhabits the nasopharynx. ${ }^{6}$ The absence of anatomical confines in nasopharyngeal tumours allows its spread via this particular method, decreasing the effectiveness of surgical management. ${ }^{14}$ Evidence for meningeal involvement can be evaluated by assessing cranial nerve functions and signs of raised intracranial pressure. ${ }^{6}$ Less than onequarter of the patients with nasopharyngeal RMS have apparent distant metastatic disease at diagnosis, with $>50 \%$ of these patients having only a single site of metastatic disease, typically in the lung. ${ }^{14}$ Haematogenous spread of the tumour has a preference for the bone marrow and lungs. ${ }^{6}$ Another unique distant site of RMS metastasis is breast tissue, with only seven formerly reported cases worldwide. ${ }^{16}$

The most significant prognostic factors affecting the outcome of patients with RMS are the age of the patient, site of the tumour, stage, and pathological subtype. Patients $>10$ years or $<1$ year of age have a worse prognosis. In contrast to other tumour locations, parameningeal RMS has the worst prognosis. ${ }^{5}$ When considering histological subtypes, the alveolar subtype is notorious for metastatic disease, leading to an 
unfavourable prognosis compared to other types. A relapsing disease of RMS has a bleak survival rate that ranges from $5-15 \% .^{5}$

Before the introduction of antineoplastic drugs, the main modality of treatment for RMS was surgery with poor survival rates, i.e., 25\% 5-year survival. ${ }^{5}$ Thereafter, the introduction of multiagent chemotherapy protocols resulted in a significant increase in long-term survival rates i.e., $70 \%$ 5-year survival. ${ }^{5}$ The present-day treatment regime of RMS includes chemotherapy, radiotherapy, and surgical management. ${ }^{17}$

The commonly chosen radiotherapy technique for paediatric RMS has originated from the Children's Oncology Group (COG) trials ARST 0331, ARST 0431, and ARST 0531, respectively. ${ }^{14}$ Treatment is given according to the planning target volume (gross tumour volume $+1 \mathrm{~cm}=$ clinical target volume). ${ }^{14}$ The planning target volume may change in accordance with the anatomy and normal tissue endurance, especially organs at risk. The inclusion of lymph nodes depends on their pathological status. This method recommends radiotherapy doses of 50.4 gray in 28 fractions at 1.8 gray per fraction to the isocentre, using $6 \mathrm{MV}$ photons and a CT scanoutlined plan.14 Treatment should be done daily, 5 days per week in a total of 5.5 weeks ${ }^{4}$ and external beam radiotherapy is an essential part of therapy. The radiation field covers the nasopharynx and neck completely. In paediatric patients, the dose is dependent on the patient's age, and other significant factors include dose per fraction, total dose, percentage volume of organ receiving dose, chemotherapy, surgery, and comorbidities i.e., hydrocephalus and diabetes.

With increasing benefits and the use of radiotherapy, long-term use complications are becoming more apparent. ${ }^{14}$ Complications of radiotherapy include sensorineural deafness, endocrine manifestations, cranial nerve palsies, cataracts, retinopathy, growth disturbance, and occurrence of secondary malignancies within the radiation field. Morbidity from radiotherapy may be astronomical and varies on the frequency and dose of radiation. Dental abnormalities are a major concern for long-term survivors, including microdontia, hypodontia, and xerostomia. Current approaches in radiotherapy are aimed at enhancing the rate of tumour control and reducing overall complications.
The administration of radiotherapy has become better with the use of CT and MRI, producing far greater image resolutions. ${ }^{14,18}$ Concomitant use with MRI improves the precision of radiotherapy, as MRI can better depict soft tissues and oedema. Radiation is more efficacious and less harmful if it is delivered conforming to the shape of the tumour. Intensity-modulated radiation therapy is based on the guidelines of conformation and converging higher radiation doses to regions within the tumour while reducing the dose to surrounding physiological critical structures. ${ }^{14}$ Regardless of the tumours' location and stage, every patient with RMS will receive chemotherapy at some point in the treatment course as a fundamental element of treatment. ${ }^{5}$ Although debate exists about the optimal chemotherapy regimen, the most important difference in treatment strategy relates to the technique and timing of local treatment. Complete surgical resection with negative margins grants the best chance of controlling local disease and decreases the local relapse rate, recuperates the overall survival, and may help avoid radiotherapy altogether. ${ }^{5}$

There can be many comparisons drawn between the presented case and the previous scientific literature. ${ }^{7-9,12,15,16}$ The major consideration is the location of the tumour, its characteristic immunohistochemistry, and bone marrow invasion of tumour cells, in this case leading to cytopenia. As was also evidently effective in two previous case studies, VAC protocol chemotherapy for metastatic RMS was administered to the patient. 8,9 The patient had RMS in the head and neck region (i.e., nasopharyngeal RMS), which are exceedingly rare, with poor prognosis. ${ }^{3}$ Hence, it should be considered as a separate clinical entity and require distinct management from that of paediatric patients because there are possible discrepancies between RMS in adults and children. ${ }^{19}$ Major histological subtypes include embryonal, alveolar, pleomorphic, and spindle cell/sclerosing RMS, which was traditionally included as a variant of embryonal but it is now considered as a separate spindle cell/sclerosing RMS subtype in the latest World Health Organization (WHO) classification (2017), and the botryoid is considered a variant. ${ }^{20}$ 


\section{CONCLUSION}

a clue for the metastatic disease. Further work-up was carried out and the patient was

The described case showcased a rare malignancy; the patient presented with nonspecific complaints of anal fissure and was diagnosed incidentally on a pelvis MRI with diagnosed imminently with nasopharyngeal RMS. The unusual site and age of the patient contributed to the novelty of this case, with a guarded prognosis.

\section{References}

1. Zhu J et al. Computed tomography and magnetic resonance imaging observations of rhabdomyosarcoma in the head and neck. Oncol Lett. 2014;8(1):155-60.

2. Canalis R et al. Nasopharyngeal rhabdomyosarcoma. A clinica perspective. Arch Otolaryngol. 1978:104(3):122-6.

3. El-Ghazali A, Mclaren KM. Embryonal rhabdomyosarcoma of adult nasopharynx. J Laryngol Otol. 2005;119(8):639-42.

4. Mondal P et al. Rhabdomyosarcoma of nose, nasopharynx and paranasal sinuses. Indian J Otolaryngol Head Neck Surg. 2009;61(4):317-9.

5. Gradoni P et al. Clinical outcomes of rhabdomyosarcoma and Ewing's sarcoma of the head and neck in children. Auris Nasus Larynx. 2011;38(4):480-6.

6. Eavey $\mathrm{R}$ et al. Rhabdomyosarcoma of the nasopharynx. Ann Otol Rhinol Laryngol. 1982;91(2 Pt 1):230-1.

7. Wight $\mathrm{R}$ et al. Rhabdomyosarcoma of the nasopharynx a case with recurrence of tumour after 20 years. $J$ Laryngol Otol. 1988;102(12):1182-4.
8. Huang $Y$ et al. Rhabdomyosarcoma of adult nasopharynx: a case report. 2005:16:146-50.

9. Wojcikiewicz E et al [Rhabdomyosarcoma of the nasopharynx--a case report] Otolaryngol Pol. 2004;58(3):631-4 (In Polish).

10. Kanagalingam $\mathrm{J}$ et al. Rhabdomyosarcoma of the adult nasopharynx. ORL J Otorhinolaryngol Relat Spec. 2002;64(3):233-6.

11. Nayar R et al. Rhabdomyosarcoma of the head and neck in adults: study of 26 patients. Laryngoscope. 1993;103(12):1362-6

12. Shindler $\mathrm{K}$ et al. Long-term followup and prognosis of orbital apex syndrome resulting from nasopharyngeal rhabdomyosarcoma. Am J Ophthalmol. 2005;140(2):236-41

13. St. John E, Woo Z Rhabdomyosarcoma of the nasopharynx. Radiology. 1955:65(2):218-22.

14. Healy J, Borg M. Paediatric nasopharyngeal rhabdomyosarcoma: a case series and literature review.
J Med Imaging Radiat Oncol. 2010;54(4):388-94.

15. Abali $\mathrm{H}$ et al. Laryngeal involvement of rhabdomyosarcoma in an adult: case report. World J Surg Oncol. 2003;1:17.

16. Beattie $M$ et al. Nasopharyngeal rhabdomyosarcoma presenting as a breast mass. Pediatr Hematol Oncol. 1990;7(3):259-63.

17. Ferrari A et al. Rhabdomyosarcoma in adults. A retrospective analysis of 171 patients treated at a single institution. Cancer. 2003:98(3):571-80.

18. Lloyd R et al. Embryonal rhabdomyosarcoma in adults. Cancer. 1983:51(3):557-65.

19. Khosla D et al. Adult rhabdomyosarcoma: clinical presentation, treatment, and outcome. J Can Res Ther 2015;11(4):830-4.

20. Sarradin $\mathrm{V}$ et al. [WHO classification of head and neck tumours 2017: main novelties and update of diagnostic methods]. Bull Cancer. 2018:105(6):596-602. (In French). 